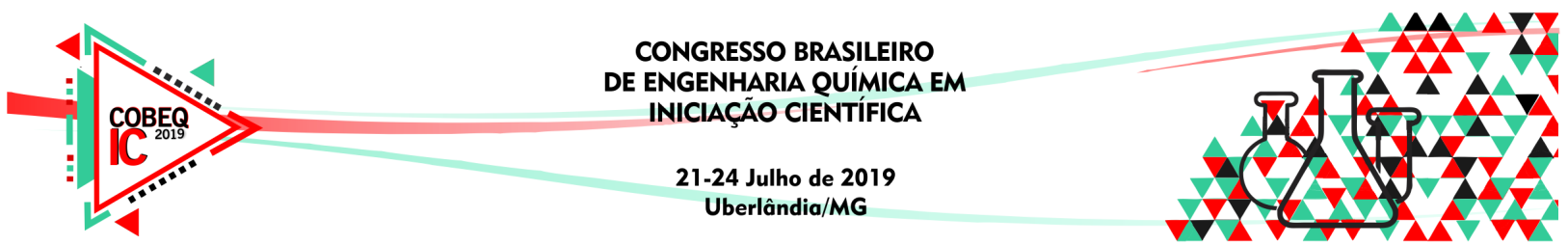

\title{
CARACTERIZAÇÃO DA FIBRA DE Calotropis procera PARA A AVALIAÇÃO DO SEU POTENCIAL ADSORTIVO
}

\section{B. B. BROMBINI ${ }^{1}$, A. K. S. XAVIER ${ }^{2}$, G. R. FERREIRA ${ }^{3}$ e J. V. W. SILVEIRA $^{4}$}

${ }^{1}$ Universidade Federal dos Vales do Jequitinhonha e Mucuri, Instituto de ciência e tecnologia ${ }^{2}$ Universidade Federal dos Vales do Jequitinhonha e Mucuri, Instituto de ciência e tecnologia

${ }^{3}$ Universidade Federal dos Vales do Jequitinhonha e Mucuri, Instituto de engenharia ciência e tecnologia

${ }^{4}$ Universidade Federal dos Vales do Jequitinhonha e Mucuri, Instituto de ciência e tecnologia E-mail para contato: joao.silveira@ict.ufvjm.edu.br

RESUMO - A Calotropis procera, planta considerada invasora pela sua facilidade de dispersão, possui em seu interior sementes que são envoltas por uma fibra que possuem aspectos hidrofóbicos, cuja o seu potencial foi observado pelos autores deste trabalho. A $C$. procera tem grande relevância uma vez que é produzida por uma planta de baixa demanda hídrica e em região pouco desenvolvida. Desta forma a fibra foi caracterizada com o intuito de ser utilizada como adsorvente. Para a caracterização da fibra foi utilizada a fibra na sua forma bruta e tratada, onde a fibra foi imersa em clorofórmio durante três dias. Após a preparação das amostras, as fibras foram caracterizadas por meio de microscopia eletrônica de varredura (MEV) e espectroscopia de absorção no infravermelho (FTIR). No MEV a fibra tratada e bruta apresenta a sua estrutura interna oca e a sua superfície lisa e no FTIR a fibra é observado a presença de hidroxilas, carbonilas alqueno aromático, entre outros, a diferença entre a fibra tratada e a bruta se dá pela presença de alcinos e alcenos que não foram encontrados na fibra bruta. Estudos posteriores serão realizados para a comprovação do potencial da $C$. procera como meio adsorvente para remoção de compostos lipofílicos, como o óleo de cozinha.

\section{INTRODUÇÃO}

Nos últimos dois séculos, o desenvolvimento da sociedade industrial e o crescimento da população têm acarretado impactos ambientais sem precedentes. Como exemplo destes impactos, ambientalistas apontam o óleo vegetal como uma das substâncias de maior potencial poluidor dos recursos naturais (SALLES, 2010). O derramamento de óleos vegetais quando realizado em cursos de água, causam grandes consequências ao meio ambiente, pois promove a formação de uma película sobre a água que dificulta a absorção de oxigênio e, por conseguinte, a destruição das algas e plânctons, além de prejudicar a sobrevivência da fauna. Frente a isso, medidas para sanar e prevenir esses acontecimentos se mostram importantes na área ambiental (MACÊDO, 2006).

Nesse contexto, considera-se importante para remediar a situação a investigação de materiais biodegradáveis, de baixo custo, fácil manejo e densidade menor que a água na remoção de óleos destas fontes hídricas. Diante disso, intensificou-se o estudo da aplicação de 


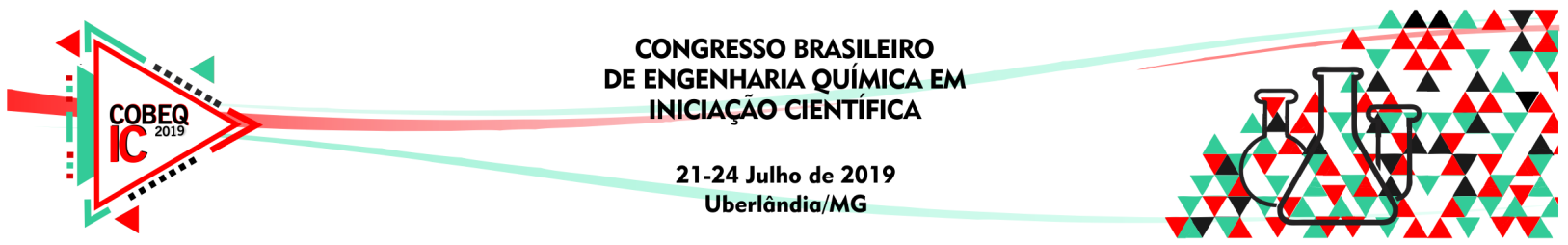

fibras naturais como materiais adsorventes para aplicação desta problemática. O estudo realizado por Vicentin (2017) mostra a utilização de fibras naturais de bucha vegetal (Luffa cylindrica) para o processo de adsorção de corante têxtil por meio de solução sintética do Laranja Reativo (BF-GR) da empresa Texpal Química (Valinhos, SP). A pesquisadora caracterizou a fibra por análise proximal, determinação de umidade, matéria volátil, cinzas, fibras, ponto de carga zero (pHpcz) e microscopia eletrônica de varredura (MEV), além dos estudos cinéticos, termodinâmicos e de equilíbrio de adsorção. Assim, Vicentin concluiu que a fibra é eficiente para o processo de adsorção do corante Laranja Reativo (BF-GR).

Ademais, estudos feitos por Aguiar et al. (2017), retrataram a análise de adsorção das fibras do mesocarpo do coco, bagaço de cana-de-açúcar e bucha vegetal para tratamento de efluentes contaminados com corantes. Inicialmente os pesquisadores testaram o corante azul de metileno em diferentes tempos e diferentes $\mathrm{pH}$ da solução, com o intuito de descobrir qual dos adsorventes apresentariam melhor desempenho. Assim, com os resultados obtidos o processo de adsorção foi aplicado no efluente da lavanderia da região de Itaperuna-RJ. Os níveis de corante após o processo de adsorção foram medidos através do valor da absorbância pelo equipamento de espectrofotometria UV-Vis. As análises mostraram que o bagaço de cana-de-açúcar e o mesocarpo do coco foram os adsorventes com maior potencial, para remover corantes dos efluentes.

A planta de Calotropis procera, conhecida popularmente como "ciúme", "algodão de seda" ou "janaúba", é nativa da Ásia e pode ser encontrada em diversos lugares do mundo como México, América Central, Austrália e América do Sul (RAHMAN e WILCOCK, 1991). Sua chegada ao Brasil se deu por volta do século XX e era utilizada como planta ornamental, mas devido a sua facilidade de adaptação em lugares degradados quimicamente passou a ser considerada uma planta invasora propagando-se em passeios, calçadas e terrenos baldios (ANDRADE et al., 2005). Pode ser aplicada em diversos segmentos como na área farmacêutica na produção de laxantes, alimentação de animais e é bastante estudada para o tratamento de doenças cardiovasculares. Como características, essa planta apresenta um fruto globuloso que internamente é preenchido com sementes junto a uma fibra de coloração branca, que são liberadas ao meio ambiente ao longo do ano (ABBAS; EL TAYEB; SULLEIMAN, 1992).

Recentemente, Morsy (2017) que realizou um estudo desta planta utilizando os caules, folhas, frutos, látex, flores e raízes e detectou em todas suas estruturas, através de técnicas como cromatografia gasosa, HPLC e UV-visível, compostos químicos como glicosídeos cardíacos e materiais lipossolúveis (esteróis, hidrocarbonetos e ácidos graxos). Através de suas análises, obteve-se o dodecano como principal hidrocarboneto presente, principalmente em flores e frutos. Já os ácidos graxos foram encontrados em todos os segmentos da planta, destacando-se os ácidos palmítico, oleico e linoleico.

Assim como Vicentin (2017) e Aguiar et al. (2017) que utilizam fibras como material adsorvente, o presente trabalho busca entender o potencial da fibra $C$. procera como material adsorvente. Entretanto diferente dos autores citados, os autores deste trabalho observaram o potencial da fibra como material hidrofóbico, ou seja, não há compatibilidade com a água. Assim, o presente trabalho realizou a caracterização da fibra de Calotropis procera para que 


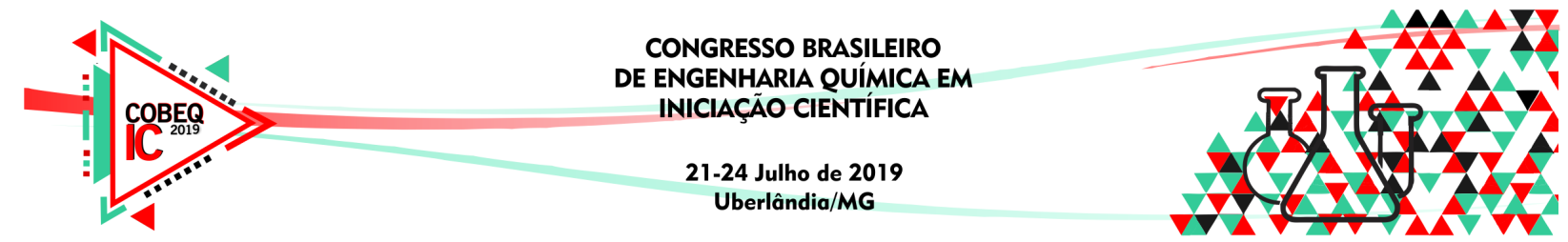

posteriormente seja analisado o seu potencial de material adsorvente utilização de remoção de óleo em água.

\section{METODOLOGIA}

\subsection{Preparação das amostras de Calotropis procera}

O procedimento inicial consistiu na preparação das amostras para serem utilizadas no decorrer deste trabalho. Para a primeira amostra, utilizaram-se as fibras de Calotropis procera bruta, sem nenhum tratamento. Posteriormente, para a segunda amostra, imergiu-se $2 \mathrm{~g}$ de fibras sem tratamento em $100 \mathrm{~mL}$ de clorofórmio durante três dias e depois deste tempo foi retirada deste líquido. A título de ilustração as amostras são apresentadas na Figura 1.

Figura 1 - Fotografias das fibras (A) Fibra sem tratamento; (B) Fibras com tratamento.

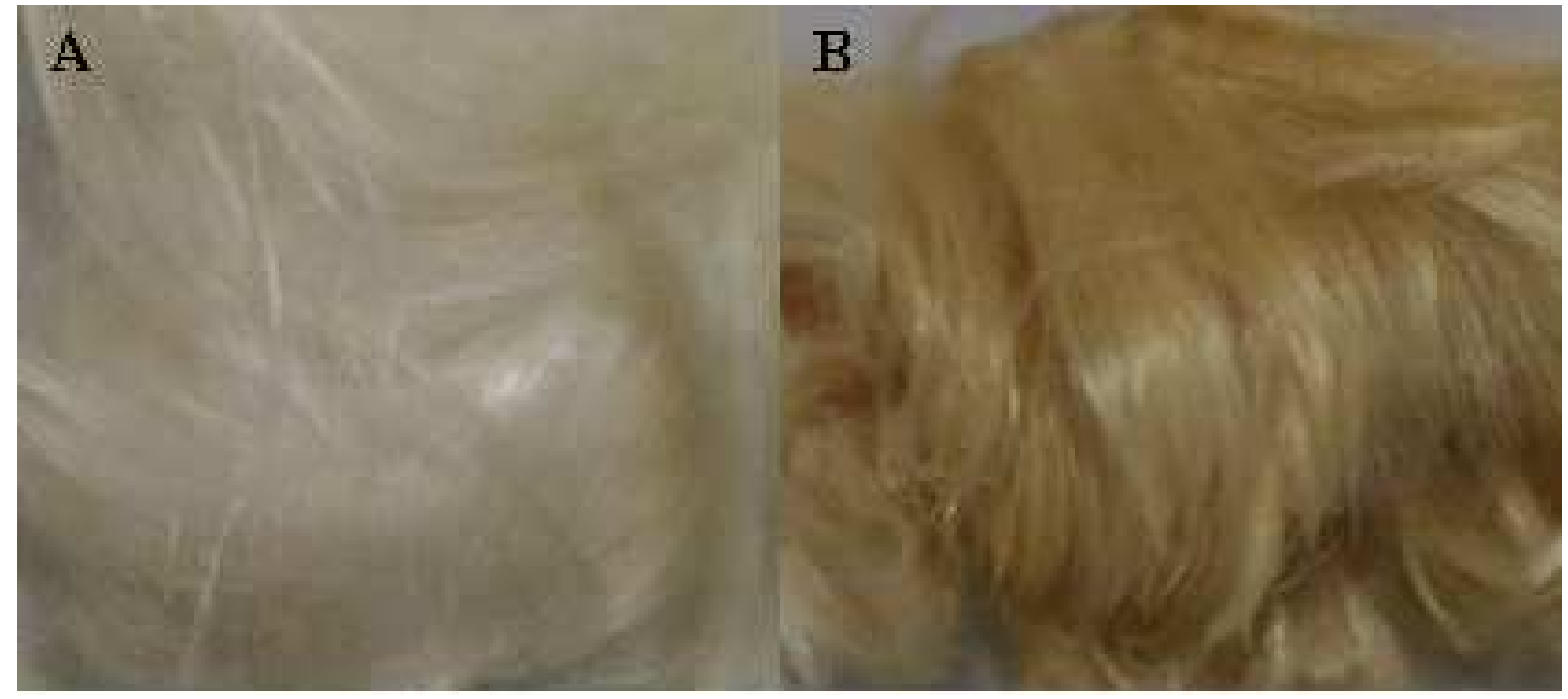

\subsection{Caracterização das fibras de Calotropis procera}

Após a preparação das amostras, as fibras foram caracterizadas por meio de microscopia eletrônica de varredura e espectroscopia de absorção no infravermelho - FTIR. Estas caracterizações serão detalhadas a seguir.

Microscopia eletrônica de varredura (MEV): a superfície da fibra foi observada no microscópio eletrônico de varredura HITACHI modelo TM 3000 Tabletop Scanning Electron Miroscope. Para a realização desta análise, cortou-se aproximadamente $0,5 \mathrm{~cm}^{2}$ das duas amostras da fibra que foram colocadas corretamente no microscópio. As imagens foram obtidas com aumentos de 50x, 100x, 1000x e 5000x. As análises foram realizadas no Laboratório de Pesquisas e Pós-Graduação LabVale na UFVJM - Campus JK.

Espectroscopia de absorção na região do infravermelho com transformada de Fourier (FTIR): os espectros de absorção na região do infravermelho com transformada de Fourier da 


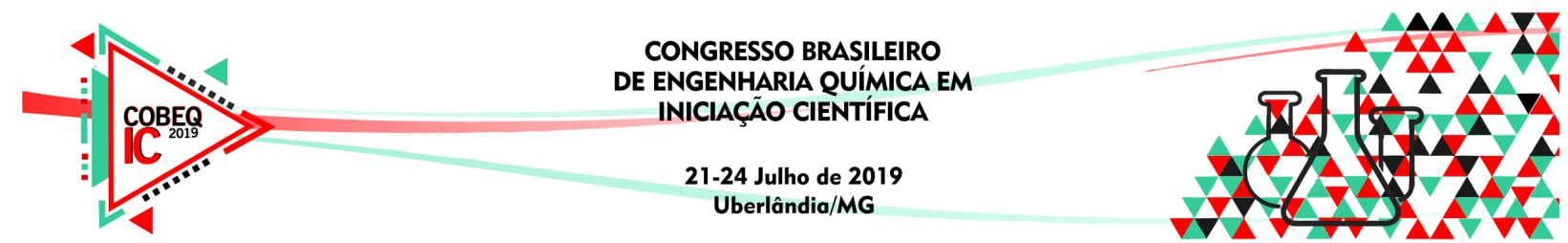

fibra de Calotropis procera foram obtidos utilizando um espectrômetro Varian, modelo 640, operando na faixa de 500 a $4000 \mathrm{~cm}^{-1}$. A caracterização foi realizada com as duas amostras descritas anteriormente e as análises foram realizadas no Laboratório de Pesquisas e PósGraduação da UFVJM - Campus JK.

\section{RESULTADOS E DISCUSSÃO}

Os resultados das caracterizações das fibras de Calotropis procera por microscopia eletrônica de varredura e espectroscopia de infravermelho das duas amostras descritas na metodologia são apresentados nesta sequência a seguir.

\subsection{Microscopia eletrônica de varredura}

As micrografias eletrônicas das fibras sem tratamento e com o tratamento nos aumentos de 50x, 100x, 1000x e 5000x conforme mostra a Figura 2.

Figura 2- Micrografias das fibras (a) sem tratamento (b) com tratamento.

(a) Sem tratamento
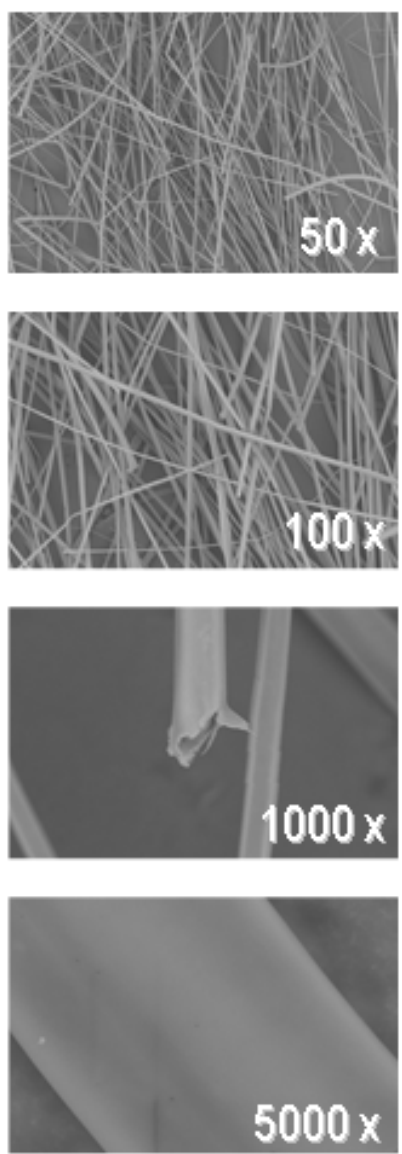

(b) Com tratamento
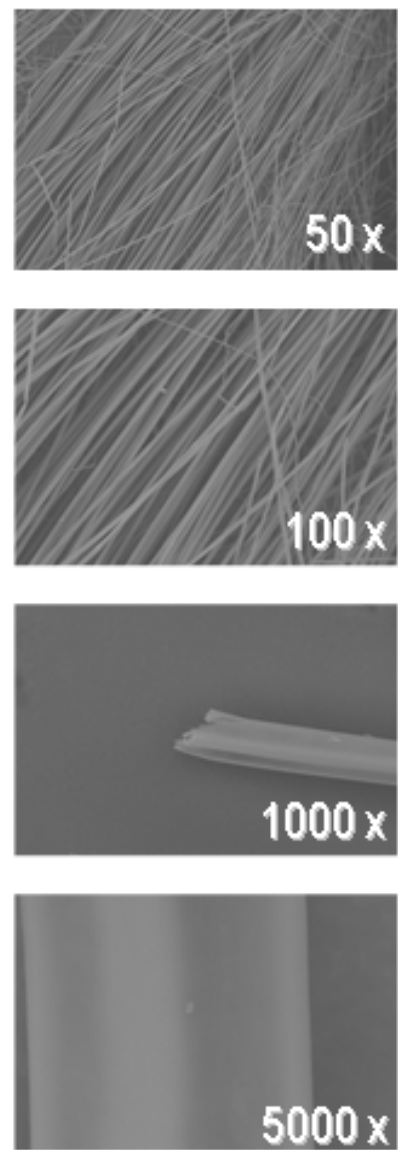


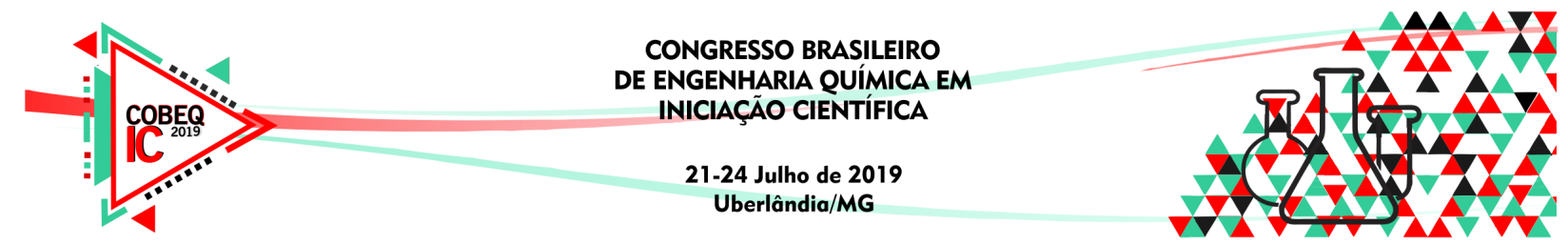

As micrografias das fibras sem tratamento e com o tratamento apresentadas na Figura 2, mostram que no o aumento de 1000x que o interior da fibra é oco e permanece oco mesmo com o tratamento realizado na mesma, fazendo com que haja uma grande área superficial. Sua superfície, como mostrada no aumento de 5000x é lisa em ambos os tratamentos realizados. A microscopia eletrônica de varredura permite ver de forma clara a estrutura da fibra analisada.

\subsection{Espectroscopia de absorção na região do infravermelho - FTIR}

A espectroscopia de absorção na região do infravermelho é uma análise utilizada para a identificação de grupos funcionais e de diferentes tipos de ligações químicas. A Figura 3 apresenta o espectro obtido para as fibras sem tratamento, para as fibras com tratamento por essa técnica.

Figura 3 - Espectrograma de FTIR das fibras com e sem tratamento.

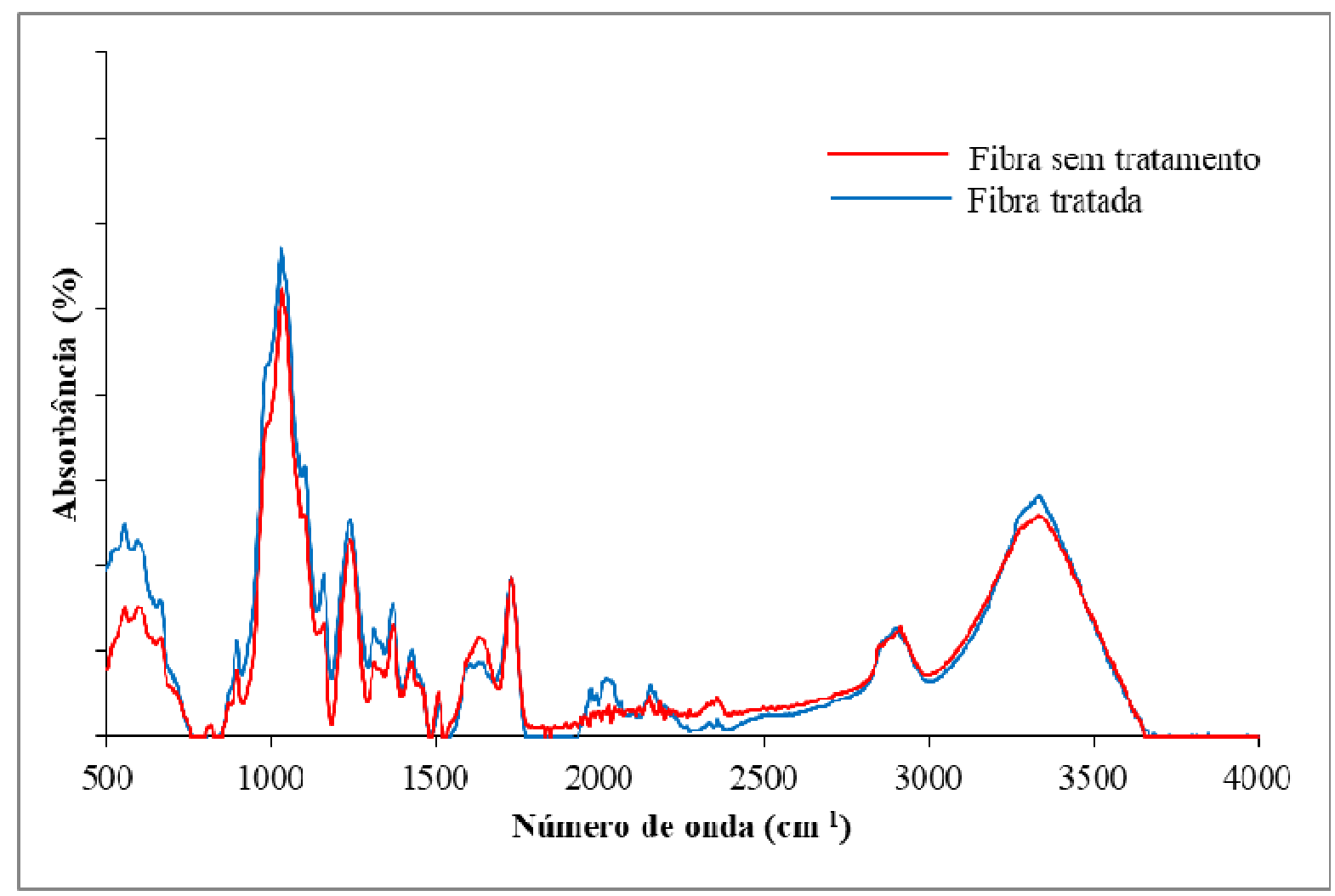

Observa-se que para a fibra sem tratamento, as principais vibrações estão localizadas em $3350 \mathrm{~cm}^{-1}, 2920 \mathrm{~cm}^{-1}$, três vibrações entre 2000 a $1500 \mathrm{~cm}^{-1}$ sendo $1730 \mathrm{~cm}^{-1}, 1640 \mathrm{~cm}^{-1} \mathrm{e}$ $1520 \mathrm{~cm}^{-1}$, posteriormente, $1380 \mathrm{~cm}^{-1}, 1320 \mathrm{~cm}^{-1}, 1250 \mathrm{~cm}^{-1}, 1040 \mathrm{~cm}^{-1}, 945 \mathrm{~cm}^{-1}$ e $820 \mathrm{~cm}^{-1}$. Segundo CELINO et al. (2014), na região do espectro entre 3000 a $3500 \mathrm{~cm}^{-1}$ nota-se características da presença de ligações $\mathrm{OH}$, explícitas no espectro em questão na vibração de $3350 \mathrm{~cm}^{-1}$. Posteriormente, em $2920 \mathrm{~cm}^{-1}$, destaca-se ligação $\mathrm{C}-\mathrm{H}$, que podem ser advindas de várias bandas devido aos estiramentos de ligações $\mathrm{C}-\mathrm{H}$ de grupos $\mathrm{CH}_{3}, \mathrm{CH}_{2}$ e $\mathrm{CH}$ de alcanos e de grupos alquilas. Na faixa de absorção entre 2000 a $1500 \mathrm{~cm}^{-1}$, a banda localizada em $1730 \mathrm{~cm}^{-1}$ está relacionada à presença de carbonilas, e as bandas agudas localizadas em 


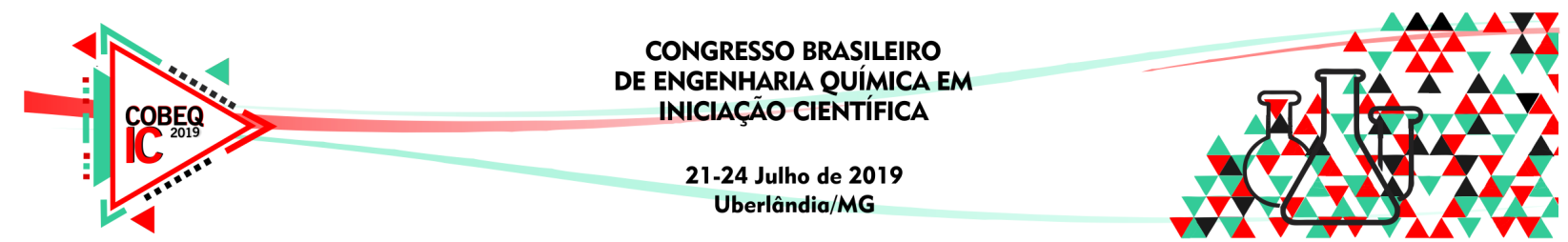

$1640 \mathrm{~cm}^{-1}$ e $1520 \mathrm{~cm}^{-1}$ indicam a presença um alqueno aromático $\mathrm{C}=\mathrm{C}$. $\mathrm{O}$ pico de $1380 \mathrm{~cm}^{-1}$, por sua vez, é relacionado a grupos $\mathrm{CH}_{3}$, em $1250 \mathrm{~cm}^{-1}$ e $1040 \mathrm{~cm}^{-1}$ as ligações $\mathrm{C}-\mathrm{O}$, típica de éteres, álcoois, fenóis, ácidos graxos, ésteres e anidridos. Comparando a espectroscopia da fibra sem tratamento com a espectroscopia da fibra com o tratamento, nota-se diferença na região do espectro entre $1750 \mathrm{~cm}^{-1}$ a $2450 \mathrm{~cm}^{-1}$. Nas fibras com o tratamento constata-se a presença de alcinos e alcenos o que não aparecem na fibra não tratada (BARBOSA, 2007).

\section{CONCLUSÃO}

Pelas caracterizações realizadas ficou claro que a fibra possui características compatíveis com materiais orgânicos, como as de óleo vegetal. Isso se deve ao fato do óleo e a fibra possuírem funções orgânicas semelhantes. Além disso, sua estrutura oca aumenta significativamente a área superficial das fibras por unidade de massa. Característica essa que potencializa sua utilização como material adsorvente. Diante disto novos testes serão realizados com o intuito de avaliar a capacidade da fibra de adsorver óleo em meio aquoso. Sendo assim, os autores agradecem ao apoio da FAPEMIG, CNPQ, UFVJM e ao apoio da LMMA patrocinada pela FAPEMIG (CEX-112-10), SECTES/MG e RQ-MG (FAPEMIG: CEX-RED-00010-14).

\section{REFERENCIAS}

ABBAS, B.; EL-TAYEB.; SULLEIMAN, Y.R.

Calotropis procera: feed potencial for arid zones. Veterinary-Record. 1992. v. 131, n. 6, p. 132.

AGUiAR, M. A. S. N.; NOLASCO, M. E. A.; SOUZA, M. R. M.; CARVALHO, J. V. S. Análise de adsorção das fibras do mesocarpo do coco, bagaço de cana-de-açúcar e bucha vegetal para tratamento de efluentes. II CONINF - Campus Itaperuna Tema "Educação em debate: novos desafios em um cenário de mudanças", 2017.

ANDRADE, M.V.M.

Aspectos fenológicos, produtivo e qualitativo da Flor de Seda. Areia, PB: Universidade Federal da Paraíba - UFPB, 2005, 82p. Dissertação (Mestrado em Zootecnia) - Centro de Ciências Agrárias / Universidade Federal da Paraíba, 2005.

BARBOSA, L. C. A.

Espectroscopia de absorção no infravermelho na caracterização de compostos orgânicos. - Viçosa: Ed. UFV, 2007.

CÉLINO, A.; GONÇALVES, O.; JACQUEMIN, F.; FRÉOUR, S.

Qualitative and quantitative assessment of water sorption in natural fibres using ATRFTIR spectroscopy. Carbohydrate Polymers, 2014. v. 101, n. 1, p. 163-170.

MACÊDO, J. A. B.

Introdução a Química Ambiental / Química; Meio Ambiente; Sociedade. 2a Edição. ed. CRQ-MG: JORGE MACEDO, 2006. 
MORSY, N.

Quantitative estimations of the abundant constituents of Calotropis procera. Main Group Chemistry, 2017. v. 16, p. 17-26.

RAHMAN, M. A.; WILCOCK, C. C.

A taxonomic revision of Calotropis (Asclepiadaceae). Nord J Bot. 1991. 11: 301 -308.

\section{SALLES, F. S. F.}

Impacto Ambiental Causado por Óleo Vegetal. 2010. 30f. Monografia - Universidade Candido Mendes, Rio de Janeiro, 2010.

\section{VICENTIN, B. .}

Estudo da adsorção de corante têxtil em fibras naturais de bucha vegetal (luffa cylindrica). Trabalho de conclusão de curso, Pato Branco - PR, 2017. 